\title{
Associated Spaces Defined by Ordinary Differential Equations
}

\author{
Le Thu Hoai and Wolfgang Tutschke
}

\begin{abstract}
The paper deals with initial value problems for desired functions $u(t, x)$ depending on the time $t$ and one spacelike variable $x$. In case the initial function $\varphi(x)$ satisfies an associated (ordinary) differential equation, the solution $u(t, x)$ satisfies the associated differential equation for each $t$. If the general solution of the associated differential equation is known, one gets a system of ordinary differential equations for the desired coefficients depending on $t$. In any case, the solution can be obtained as fixed point of a related integro-differential operator.
\end{abstract}

Keywords. Initial value problem, contraction-mapping principle, separation of variables, conservation law

Mathematics Subject Classification (2000). Primary 35A05, secondary 35G10, $35 \mathrm{G} 25$

\section{Statement of the problem}

Solutions of the initial value problem

$$
\begin{aligned}
\partial_{t} u & =\mathcal{F}\left(t, x, u, \partial_{x} u, \ldots, \partial_{x}^{k} u\right) \\
u(0, x) & =\varphi(x),
\end{aligned}
$$

(where $t$ means the time and $x$ is a single real variable) are fixed points of the operator

$$
U(t, x)=\varphi(x)+\int_{0}^{t} \mathcal{F}\left(\tau, x, u, \partial_{x} u, \ldots, \partial_{x}^{k} u\right) d \tau .
$$

If the initial function $\varphi$ satisfies an associated equation $\mathcal{G} \varphi=0$, then there exists a (uniquely determined) solution also satisfying the associated equation for each $t$. A pair $\mathcal{F}, \mathcal{G}$ (where the coefficients of $\mathcal{G}$ do not depend on $t$ ) is said to be associated if $\mathcal{G} u=0$ implies $\mathcal{G}(\mathcal{F} u)=0$ for each $t$.

Le Thu Hoai: Hanoi University of Technology, Faculty of Applied Mathematics and Informatics, Dai Co Viet Road 1, 10000 Hanoi, Vietnam; hoailethu@yahoo.com W. Tutschke: Graz University of Technology, Department of Mathematics D, Steyrergasse 30/3, A-8010 Graz, Austria; tutschke@tugraz.at 


\section{Estimation of the integro-differential operator}

Let $I=\left\{x: x_{1} \leq x \leq x_{2}\right\}$ be a bounded and closed interval on the $x$ axis. Let $\mathcal{B}(I)$ the Banach space of functions $u$ which are $k$ times continuously differentiable in $I$ equipped with the supremum norm

$$
\|u\|=\max \left(\sup _{I}|u|, \sup _{I}\left|u^{\prime}\right|, \ldots, \sup _{I}\left|u^{(k)}\right|\right)
$$

(if $I$ is not closed and/or not bounded, $\mathcal{B}(I)$ is the space of $k$ times continuously differentiable functions $u$ for which $u$ and the derivatives up to the order $k$ are bounded). Consider the rectangle $M_{T}=I \times[0, T]$, where $T$ will be fixed later.

Now let $\mathcal{B}_{*}\left(M_{T}\right)$ be the Banach space of functions $u(t, x)$ defined and continuous in $M_{T}$ such that $\|u(t, \cdot)\|$ is finite for each $t$. Define

$$
\|u\|_{*}=\sup _{0 \leq t \leq T}\|u(t, \cdot)\|
$$

Suppose $\mathcal{G}$ is a linear and homogeneous ordinary differential operator defined in $I$,

$$
\mathcal{G} u \equiv u^{(k)}-a_{1}(x) u^{(k-1)}-\ldots-a_{k}(x) u=0,
$$

whose coefficients are supposed to be $k$ times continuously differentiable. Consider the subspace $\mathcal{B}_{*}^{\mathcal{G}}\left(M_{T}\right)$ of $\mathcal{B}_{*}\left(M_{T}\right)$ containing all functions $u(t, x)$ which satisfy the differential equation $\mathcal{G} u(t, \cdot)=0$ for each $t$. For functions $u(t, x)$ belonging to $\mathcal{B}_{*}^{\mathcal{G}}\left(M_{T}\right)$, the derivative of order $k$ can be replaced by the lower order derivatives. Therefore we have in the integrand of (3) only derivatives of $u$ up to the order $k-1$. Provided the right-hand side $\mathcal{F}\left(t, x, u, p_{1}, \ldots, p_{k}\right)$ is $k$ times continuously differentiable with respect to $x, u, p_{1}, \ldots, p_{k}$ (and the initial function $\varphi$ is also $k$ times continuously differentiable), the image $U(t, x)$ can be differentiated with respect to $x$ under the sign of integration. That way one obtains a representation of $\partial_{x} U$ by an integral whose integrand contains $u$ and its derivatives $\partial_{x}^{j} u$ up to the order $k$. Again we can replace $\partial_{x}^{k} u$ by lower order derivatives, and so $\partial_{x}^{2} U$ exists. Repeating this construction we see, finally, that $U$ has derivatives with respect to $x$ up to the order $k$. All these derivatives can be represented by integrals whose integrands contain only derivatives of $u$ up to the order $k-1$.

Additionally we assume that $\mathcal{F}$ and its derivatives mentioned above satisfy a Lipschitz condition with repect to $u, p_{1}, \ldots, p_{k}$, that is

$$
\begin{aligned}
\mid \mathcal{F}\left(t, x, u, p_{1}, \ldots, p_{k}\right) & -\mathcal{F}\left(t, x, v, q_{1}, \ldots, q_{k}\right) \mid \\
& \leq L_{00}|u-v|+L_{01}\left|p_{1}-q_{1}\right|+\ldots+L_{0 k}\left|p_{k}-q_{k}\right| .
\end{aligned}
$$

Denote the Lipschitz constants of $\mathcal{F}$ after $i$ differentiations with respect to $x$ by $L_{i 0}, L_{i 1}, \ldots, L_{i k}, i=1, \ldots, k$. Notice that $\mathcal{F}$ and its derivatives satisfy such a 
Lipschitz condition if $\mathcal{F}$ is linear in $u, p_{1}, \ldots, p_{k}$ and the coefficients and their derivatives (up to the order $k$ ) are bounded. Then the Lipschitz constants are given by bounds for the absolute value of the coefficients and their derivatives (see Example 4.1 below).

Consider two elements $u$ and $v$ of $\mathcal{B}_{*}^{\mathcal{G}}\left(M_{T}\right)$ and their images $U$ and $V$ respectively. The above integral representations of $U$ and $V$ and their derivatives and the Lipschitz conditions of type (4) for $\mathcal{F}$ and its derivatives lead to the estimate

$$
\|U-V\|_{*} \leq N \cdot \max _{i, j} L_{i j} \cdot T \cdot\|u-v\|_{*},
$$

where $N$ is the number of terms to be estimated. This shows that the operator (3) is contractive if $T$ is small enough. Generally speaking, the operator (3) does not map the space $\mathcal{B}_{*}^{\mathcal{G}}\left(M_{T}\right)$ into itself. This, however, is the case if $\mathcal{F}$ and $\mathcal{G}$ form an associated pair. Then the contraction-mapping principle can be applied, and we get a uniquely determined fixed point in $\mathcal{B}_{*}^{\mathcal{G}}\left(M_{T}\right)$.

Replacing the above norm $\|\cdot\|_{*}$ by the weighted norm

$$
\|u\|_{\sigma}=\sup _{0 \leq t \leq T}\left\|e^{-\sigma t} u(t, \cdot)\right\|
$$

one has $\left|\partial_{x}^{j} u-\partial_{x}^{j} v\right| \leq e^{\sigma t}\|u-v\|_{\sigma}$ (weighted $L_{p}$-norms are used in L. v. Wolfersdorf's and J. Janno's paper [2]). Thus in the integral representation of $\partial_{x}^{j} U-\partial_{x}^{j} V$ the additional factor $e^{\sigma \tau}$ occurs leading to

$$
\int_{0}^{t} e^{\sigma \tau} d \tau=\frac{1}{\sigma}\left(e^{\sigma t}-1\right)<\frac{1}{\sigma} e^{\sigma t}
$$

Writing $e^{\sigma t}$ as $e^{-\sigma t}$ on the left-hand side of the estimate of $\left|\partial_{x}^{j} U-\partial_{x}^{j} V\right|$, it follows

$$
\|U-V\|_{\sigma} \leq \frac{1}{\sigma} \cdot N \cdot \max _{i, j} L_{i j} \cdot\|u-v\|_{\sigma}
$$

instead of (5). Provided $\sigma$ is large enough, the operator is thus contractive for each $T$.

\section{The final result}

Summarizing the above arguments, we have proved the following conservation law:

Theorem 3.1. Suppose the operator $\mathcal{G}$ is associated to the right-hand side $\mathcal{F}$. Suppose, further, that $\mathcal{F}$ and its derivatives mentioned above are Lipschitzcontinuous. In case the ( $k$ times continuously differentiable) initial function $\varphi$ satisfies an associated differential equation and $T$ is small enough, the initial value problem under consideration possesses a uniquely determined solution $u(t, x)$ in the space $\mathcal{B}_{*}^{\mathcal{G}}\left(M_{T}\right)$. In other words, the side condition $\mathcal{G} u(t, \cdot)=0$ is satisfied for each $t$ with $0 \leq t \leq T$. 
Denote by $\mathcal{B}_{\sigma}^{\mathcal{G}}\left(M_{T}\right)$ the Banach space of functions $u$ defined in $M_{T}$ for which $\|u\|_{\sigma}$ is finite. Suppose $\mathcal{F}$ is defined for all $t$ with $0 \leq t \leq T$. Then the estimate (6) shows:

Corollary 3.2. Using the weighted norm $\|\cdot\|_{\sigma}$ with

$$
\sigma>N \cdot \max _{i, j} L_{i j}
$$

the initial value problem is solvable in $\mathcal{B}_{\sigma}^{\mathcal{G}}\left(M_{T}\right)$ without making $T$ smaller.

Note that the existence of a solution of the initial value problem with an initial function $\varphi$ satisfying $\mathcal{G} \varphi=0$ does not exclude the existence of further solutions which satisfy additionally boundary conditions. These additional solutions $u(t, x)$, however, cannot be solutions of the side condition $\mathcal{G} u(t, \cdot)=0$ for each $t$.

Provided the general solution $\sum_{j=1}^{k} \mu_{j} \varphi_{j}(x)$ of the associated differential equation $\mathcal{G} u=0$ is known, the desired solution has the same form for each $t$. This yields the following statement:

Theorem 3.3. Suppose the right-hand side $\mathcal{F}$ is linear and homogeneous in $u$ and its derivatives up to the order $k$. Suppose, further, that the initial function $\varphi$ satisfies an associated differential equation for which one knows the general solution. Then the desired solution has the form $\sum_{j=1}^{k} \mu_{j}(t) \varphi_{j}(x)$. Substituting this linear combination into the differential equation (1) and comparing the coefficients of the $\varphi_{j}(x)$, one obtains a first order system for the $\mu_{j}(t)$ whose initial values $\mu_{j}(0)$ are uniquely determined by the given initial function.

Consequently, the application of fixed-point methods is not necessary in case one knows the general solution of an associated differential equation. So the method of associated differential operators can lead to explicit solutions (see the Examples 4.4, 4.5 and 4.6).

\section{Examples and a concluding remark}

Example 4.1. Suppose $\mathcal{F}\left(t, x, u, p_{1}, p_{2}\right)=p_{2}$ and $\mathcal{G} u \equiv u^{\prime \prime}-a(x) u^{\prime}-b(x) u$. Determine the Lipschitz constants of $\mathcal{F}$ and its derivatives with respect to $x$ up to the second order if $C$ is a bound of $|a|,\left|a^{\prime}\right|,\left|a^{\prime \prime}\right|,|b|,\left|b^{\prime}\right|$ and $\left|b^{\prime \prime}\right|$.

Since the integrands for $U, \partial_{x} U$ and $\partial_{x}^{2} U$ are

$$
\begin{aligned}
& \partial_{x}^{2} u \\
& a \partial_{x}^{2} u+\left(a^{\prime}+b\right) \partial_{x} u+b^{\prime} u \\
& \left(a^{\prime}+a^{2}+b\right) \partial_{x}^{2} u+\left(a^{\prime \prime}+2 a a^{\prime}+a b+2 b^{\prime}\right) \partial_{x} u+\left(a^{\prime} b+a b^{\prime}+b^{\prime \prime}\right) u
\end{aligned}
$$


we get

$$
\begin{array}{lll}
L_{00}=0, & L_{01}=0, & L_{02}=1 \\
L_{10}=C, & L_{11}=2 C, & L_{12}=C \\
L_{20}=2 C^{2}+C, & L_{21}=3 C^{2}+3 C, & L_{22}=C^{2}+2 C,
\end{array}
$$

and we have $N=7$.

Example 4.2. The first order differential equation

$$
\partial_{t} u=\partial_{x} u+u^{2}
$$

shows that the method of associated differential equations can also be applied if the right-hand side $\mathcal{F}$ does not satisfy a global Lipschitz condition.

Indeed, $\mathcal{G} u \equiv u^{\prime}=0$ is associated to the right-hand side of (7). Consequently, constant initial functions $\varphi$ lead to a conservation law. If $\varphi(x) \equiv \lambda=$ const, then the construction of characteristics gives the solution

$$
u(t, x)=\frac{\lambda}{1-\lambda t},
$$

which is constant for each $t$.

Linear operators $\mathcal{F}$ of order $k$ with $k$ times continuously differentiable coefficients depending on $x$ possess always associated systems of order $k$ :

Example 4.3. To the right-hand side

$$
\mathcal{F} u=\sum_{j=0}^{k} A_{k-j}(x) \partial_{x}^{j} u
$$

there exist always associated operators

$$
\mathcal{G} u \equiv u^{(k)}-\sum_{j=1}^{k} a_{j}(x) u^{(k-j)}
$$

whose coefficients can be obtained from a system of ordinary differential equations.

Consider $\mathcal{G}(\mathcal{F} u)$. Using the side condition $\mathcal{G} u=0$, the derivatives of order $k$ in $\mathcal{F} u$ can be replaced by lower order derivatives. That way one gets

$$
\mathcal{G}(\mathcal{F} u)=\partial_{x}^{k}\left(A_{0} \sum_{j=1}^{k} a_{j} \partial_{x} u^{k-j}\right)-\sum_{i=1}^{k} a_{i} \partial_{x}^{k-i}\left(A_{0} \sum_{j=1}^{k} a_{j} \partial_{x} u^{k-j}\right)
$$


Again and again replacing the derivatives of order $k$ by lower order derivatives, one gets finally a linear combination of $u, \partial_{x} u, \ldots, \partial_{x}^{k-1} u$. Clearly, we have $\mathcal{G}(\mathcal{F} u)=0$ if all coefficients of this linear combination vanish identically. The resulting system has the form

$$
A_{0} a_{j}^{(k)}+\text { lower order derivatives of } a_{1}, \ldots, a_{k}=0, \quad j=1, \ldots, k,
$$

since the highest order derivatives of $a_{1}, \ldots, a_{k}$ come only from the first sum on the right-hand side of (8). Provided $A_{0} \neq 0$, this is a system of ordinary differential equations of order $k$ for $a_{1}, \ldots, a_{k}$.

Note that one gets the system (9) for the $a_{j}$ also if the $A_{k-j}$ depend not only on $x$ but also on $t$. Nevertheless, it is possible that the solutions $a_{j}$ depend only on $x$ (see Example 4.5 below).

It can happen that a system for the coefficients of an associated equation is not globally solvable in the interval $I$ but only in a subinterval $I^{\prime}$ of $I$. Then one gets a conservation law only in the subinterval $I^{\prime}$.

Generally speaking, in case the general solution of an associated differential equation is known, then the corresponding solutions can be determined by solving a system of ordinary differential equations for the coefficients of the general solution (instead of solving the equivalent integro-differential equation). This will be shown by the next three examples:

Example 4.4. The second order operator

$$
\mathcal{F}\left(t, x, u, \partial_{x} u, \partial_{x}^{2} u\right)=\partial_{x}^{2} u+A(x) u .
$$

and $\mathcal{G} \equiv u^{\prime \prime}-a u^{\prime}-b u=0$ are an associated pair if $a$ and $b$ satisfy the second order system

$$
\begin{aligned}
a^{\prime \prime}+2 a a^{\prime}+2\left(b^{\prime}+\partial_{x} A\right) & =0 \\
b^{\prime \prime}+2 a^{\prime} b+\partial_{x}^{2} A-a \partial_{x} A & =0 .
\end{aligned}
$$

Similar conditions can be obtained for the second order operator

$$
\mathcal{F}\left(t, x, u, \partial_{x} u, \partial_{x}^{2} u\right)=A_{0}(x) \partial_{x}^{2} u+A_{1}(x) \partial_{x} u+A_{2}(x) u .
$$

For the heat equation $(A \equiv 0$ in $(10))$ the associated pair has to satisfy

$$
\begin{aligned}
a^{\prime \prime}+2 a a^{\prime}+2 b^{\prime} & =0 \\
b^{\prime \prime}+2 a^{\prime} b & =0 .
\end{aligned}
$$

In particular, all equations $\mathcal{G} u \equiv u^{\prime \prime}-a u^{\prime}-b u=0$ with constant coefficients are associated to the one-dimensional heat equation. Choosing $a=-2$ and $b=-1$, one gets $\left(\mu_{1}+\mu_{2} x\right) \exp (-x)$ as general solution of the corresponding associated 
equation. Initial functions $\varphi(x)=\left(c_{1}+c_{2} x\right) \exp (-x)$ lead thus to solutions of the form $u(t, x)=\left(\mu_{1}(t)+\mu_{2}(t) x\right) \exp (-x)$. The coefficients $\mu_{1}$ and $\mu_{2}$ have to satisfy the first order system

$$
\begin{aligned}
& \mu_{1}^{\prime}=\mu_{1}-2 \mu_{2} \\
& \mu_{2}^{\prime}=\mu_{2} .
\end{aligned}
$$

This leads to the explicit solution

$$
u(t, x)=\left(\left(c_{1}-2 c_{2} t\right)+c_{2} x\right) \exp (t-x) .
$$

Explicit solutions can also be constructed by separation of variables: If $u(t, x)=\mu(t) f(x)$, then the heat equation leads to

$$
\mu^{\prime}=c \mu \text { and } f^{\prime \prime}=c f,
$$

where $c$ is constant. The differential equations $f^{\prime \prime}=c f$ are special associated differential equations $\mathcal{G} u \equiv u^{\prime \prime}-a u^{\prime}-b u=0$ with $a=0$ and $b=-c$. Therefore, the method of associated differential equations leads to more explicit solutions as the usual separation of variables does. The construction of solutions with the help of a general solution can also be interpreted as some kind of separation of variables.

Notice that for the heat equation there exist also associated differential equations with non-constant coefficients such as

$$
a(x)=k_{1} \tanh \left(k_{1}\left(x+k_{2}\right)\right), \quad b \equiv 0,
$$

where $k_{1}$ and $k_{2}$ are constants.

Example 4.5. The method of associated differential equations can also be applied to equations $\partial_{t} u=\mathcal{F} u$ whose right-hand side depends on $t$. Consider, for instance, $\partial_{t} u=\partial_{x}^{2} u+\exp (-t) u$. One of its associated equations is $u^{\prime \prime}+u=0$.

Since $\cos x$ and $\sin x$ form a general solution of the associated equation $u^{\prime \prime}+u=0$, the equation $\partial_{t} u=\mathcal{F} u$ has solutions of the form

$$
u(t, x)=\mu(t) \cos x+\nu(t) \sin x .
$$

The desired coefficients $\mu(t)$ and $\nu(t)$ have to satisfy the system

$$
\begin{aligned}
\mu^{\prime}+\mu(1-\exp (-t)) & =0 \\
\nu^{\prime}+\nu(1-\exp (-t)) & =0
\end{aligned}
$$

This leads to the solution

$$
u(t, x)=\exp (1-t-\exp (-t))\left(c_{1} \cos x+c_{2} \sin x\right)
$$

if the initial function is $\varphi(x)=c_{1} \cos x+c_{2} \sin x$. By the way, here the system (9) is again the system (11), (12), that is, the system does not depend on $t$ although $\mathcal{F}$ does. 
Example 4.6. If $\mathcal{F} u=u \partial_{x} u$ and $\mathcal{G} u \equiv u^{\prime}-a(x) u=0$, then permissible coefficients $a(x)$ have to satisfy the ordinary differential equation $a^{\prime}+a^{2}=0$. Initial functions $\varphi$ satisfying an associated differential equation have thus to be linear functions $\varphi(x)=\mu_{0}+\nu_{0} x$, where $\mu_{0}$ and $\nu_{0}$ are constants. The conservation law implies the existence of solutions of the form $u(t, x)=\mu(t)+$ $\nu(t) x$. This leads to the system

$$
\begin{aligned}
\mu^{\prime} & =\mu \nu \\
\nu^{\prime} & =\nu^{2} .
\end{aligned}
$$

Solving this system, one gets the desired solution

$$
u(t, x)=\frac{1}{1-\nu_{0} t}\left(\mu_{0}+\nu_{0} x\right) .
$$

Of course, since $\mathcal{F} u=u \partial_{x} u$ is locally Lipschitz continuous, one can also apply the contraction-mapping principle to a ball of radius $R$ centred at $u(t, x) \equiv 0$ provided $R>\max \left(\sup _{I}|\varphi|, \sup _{I}\left|\varphi^{\prime}\right|\right)$.

Remark 4.7. In case $k=1$ and $x=\left(x_{1}, \ldots, x_{n}\right), n \geq 2$, initial value problems of type (1), (2) can also be solved in associated spaces. However, the solution does exist only in conical domains (see [1] where one can find further references).

Acknowledgement. The first version of the paper did not make use of the weighted norm $\|\cdot\|_{\sigma}$. The authors are grateful to one of the referees who proposed to construct a global solution by using this norm. - The first-named author was supported by the Austrian Asea-Uninet Programme.

\section{References}

[1] Tutschke, W., Associated partial differential operators - applications to well- and ill-posed problems. In: Abstract and Applied Analysis (eds.: Nguyen Minh Chuong et al.). Singapore: World Scientific 2004, pp. 373 - 383.

[2] Wolfersdorf, L. v. and Janno, J., On a class of nonlinear convolution equations. J. Anal. Anwendungen 14, (1995)(3), $497-508$.

Received December 22, 2004; revised April 25, 2005 